\title{
Magnetic Losses Evaluation in PM SM Drive with Non Sinusoidal Supply by Finite Elements Analysis and Superposition Principle
}

\author{
R.Kaczmarek, W.-Y.Huang and J.-C. Vannier
}

\begin{abstract}
Field superposition in rotating machines with sinusoidal distribution of magneto motive force and non sinusoidal voltage supply is analyzed in view of specific behavior of field components rotating in opposite direction at the same velocity in relation to rotor. These components $-5^{\text {th }}$ and $7^{\text {th }}$, or $11^{\text {th }}$ and $13^{\text {th }}$ etc - form oscillating fields which imply rotor losses quite different than it is usually expected in superposition procedures, where overall losses are given by sum of effects produced by individual harmonic components of sliding fields. In case of eddy current losses in rotor's permanent magnets the difference for one pair of harmonics can reach several tens per cent.
\end{abstract}

Index Terms - Permanent Magnet Synchronous Machine, magnetic losses, magnetic fields, harmonic analysis

\section{NOMENCLATURE}

$i_{a}, i_{b}, i_{c}$ - armature phase currents

$n$ - conductor number per phase

$2 \mathrm{r}$ - rotor's diameter

$\mathrm{d}$ - rotor's length

$\theta$ - angle coordinates in stator reference frame

$\theta_{\mathrm{r}}$ - angle coordinates in rotor reference frame

\section{INTRODUCTION}

$\mathrm{T}^{\mathrm{s}}$ he PWM supplied permanent magnet synchronous machines are exposed to magnets' heating due to harmonic fields which rotate in relation to rotor [1]-[5]. Here the usual way of calculation of magnetic is with immobile rotor and the rotor related harmonic currents on armature side [6]-[7]. This modelling has the advantage of sparing of cumbersome and inefficient simulation of rotor in movement. With immobile rotor the PM losses can be calculated by FE simulation for each relevant rotor side frequency, the losses calculated for one frequency (the $6^{\text {th }}$, e.g.) being allocated to both generic fields rotating in opposit directions (the $5^{\text {th }}$ and the $7^{\text {th }}$ in this example), with final superposition of all the loss components.

R.Kaczmarek is with Dept. Energy of Supelec, 91192 Gif sur Yvette, France (robert.kaczmarek@supelec.fr)

J-C.Vannier is with Dept. Energy of Supelec, 91192 Gif sur Yvette, France (Jean-Claude.Vannier@supelec.fr)

W.-Y.Huang is with Dept. Energy of Supelec, 91192 Gif sur Yvette, France (wanying.huang@supelec.fr)
However, the sliding fields see the rotor's dissipating space not in the same way as oscillating fields do. Consequently, superposition of individual losses may differ substantially from losses dissipated by composed fields.

In order to fix conditions for superposition to work, we start with harmonic fields' analysis in stator and in rotor reference frame, paying particular attention to the fields' initial phases.

\section{SLIDING AND COMPOSED MMF}

\section{A. Stator reference}

In inverter-fed PM SM the armature currents are usually non sinusoidal, whereas the spatial distribution of magnetomotive force can be assumed as sinusoidal with stator reference frame fixed $(\theta=0)$ in geometrical axe of winding distribution of phase "a". This axe points to maximum of the cosine expression for MMF of phase "a" (1):

$$
\begin{aligned}
& F_{a}(\theta)=\frac{2}{\pi} n i_{a} \cos (\theta) \\
& F_{b}(\theta)=\frac{2}{\pi} n i_{b} \cos \left(\theta-120^{\circ}\right) \\
& F_{c}(\theta)=\frac{2}{\pi} n i_{c} \cos \left(\theta-240^{\circ}\right)
\end{aligned}
$$

If we start counting time from the moment the fundamental current in "a" winding is at its top

$$
\begin{aligned}
& i_{a 1}(t)=I_{1} \cos (\omega t) \\
& v_{a 1}(t)=V_{1} \cos \left(\omega t+\varphi_{1}\right)
\end{aligned}
$$

then we find top value of the fundamental composed magneto motive force at $\theta=0$

$$
F_{l}(\theta, t)=F_{a l}(\theta, t)+F_{b l}(\theta, t)+F_{c l}(\theta, t)
$$

In this moment the rotor axe is lagging the stator axe, and obviously also the fundamental MMF, by the machine internal angle $\delta$ (Fig. 1).

For further analysis we take the $5^{\text {th }}$ and the $7^{\text {th }}$ armature current harmonics (4): 


$$
\begin{aligned}
& i_{a 5}(t)=I_{5} \cos \left(5 \omega t+\phi_{5}\right) \\
& i_{b 5}(t)=I_{5} \cos \left(5 \omega t+\phi_{5}+120^{\circ}\right) \\
& i_{c 5}(t)=I_{5} \cos \left(5 \omega t+\phi_{5}+240^{\circ}\right) \\
& i_{a 7}(t)=I_{7} \cos \left(7 \omega t+\phi_{7}\right) \\
& i_{b 7}(t)=I_{7} \cos \left(7 \omega t+\phi_{7}-120^{\circ}\right) \\
& i_{c 7}(t)=I_{7} \cos \left(7 \omega t+\phi_{7}-240^{\circ}\right)
\end{aligned}
$$

with initial phases $\phi_{5}$ and $\phi_{7}$ measured in relation to their corresponding harmonic voltages, which are all in-phase with supply PWM voltage and, obviously, with its fundamental component.

These currents form a pair of fields $F_{5}, F_{7}$ of classical form of sinusoidal distribution along the air gap, rotating in relation to stator at, respectively, $-5 \omega$ :

$$
F_{5}(\theta)=\frac{3 n I_{5}}{\pi} \cos \left(5 \omega t+\phi_{5}+\theta\right)
$$

and $+7 \omega$ :

$$
F_{7}(\theta)=\frac{3 n I_{7}}{\pi} \cos \left(7 \omega t+\phi_{7}-\theta\right)
$$

At $\mathrm{t}=0$ the $F_{5}(\theta)$ has its maximum in $\theta=-\phi_{5}$ and $F_{7}(\theta)$ at $\theta=\phi_{7}$ (Fig.1).

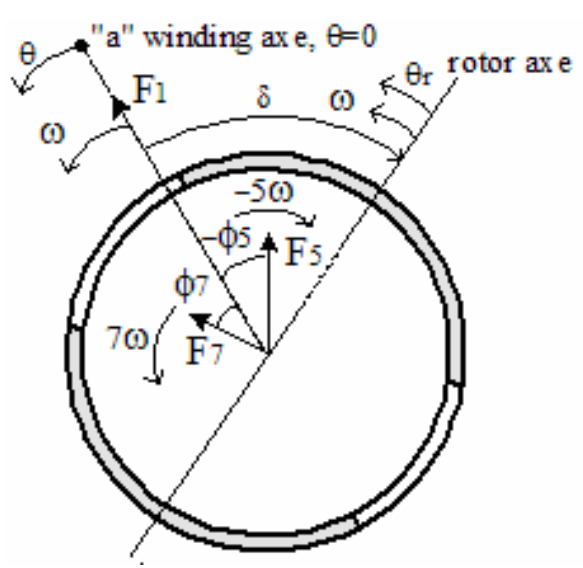

Fig. 1. The fundamental, the $5^{\text {th }}$ and the $7^{\text {th }}$ harmonics of MMF in relation to stator, represented here by its " $a$ " winding at $t=0$. Rotor is schemed for one pair of poles with magnets' indicated in black.

We should now relate all these quantities to rotor.

\section{B. Rotor reference}

In rotor reference frame we will see these fields as created by phase currents of, respectively, negative and positive sequences (7):

$$
\begin{aligned}
& i_{a-6}(t)=I_{-} \cos \left(6 \omega t+\phi_{-6}\right) \\
& i_{b-6}(t)=I_{-} \cos \left(6 \omega t+\phi_{-6}+120^{\circ}\right) \\
& i_{c-6}(t)=I_{-} \cos \left(6 \omega t+\phi_{-6}+240^{\circ}\right)
\end{aligned}
$$

$$
\begin{aligned}
& i_{a 6}(t)=I_{+} \cos \left(6 \omega t+\phi_{+6}\right) \\
& i_{b 6}(t)=I_{+} \cos \left(6 \omega t+\phi_{+6}-120^{\circ}\right) \\
& i_{c 6}(t)=I_{+} \cos \left(6 \omega t+\phi_{+6}-240^{\circ}\right)
\end{aligned}
$$

these currents circulating in virtual 3-phases windings fixed on the rotor in position of $\delta$ ahead of the rotors axe; at $\mathrm{t}=0$ this position is that of the stator " $a$ " winding. Then the rotor related MMFs have the form (8):

$$
\begin{aligned}
& F_{a 6}=\frac{2}{\pi} n i_{a 6} \cos \left(\theta_{r}-\delta\right) \\
& F_{b 6}=\frac{2}{\pi} n i_{b 6} \cos \left(\theta_{r}-\delta-120^{\circ}\right) \\
& F_{c 6}=\frac{2}{\pi} n i_{c 6} \cos \left(\theta_{r}-\delta-240^{\circ}\right)
\end{aligned}
$$

The phase $\phi$ in current expression being nothing more than initial angles, we have $\phi_{-6}=\phi_{5}$ and $\phi_{+6}=\phi_{7}$. Amplitudes don't change, neither, when passing from stator to rotor reference frame and so we have $I_{+}=I_{7}$ and $I_{-}=I_{5}$.

The magneto-motive force $F_{+6}$ resulting from positive sequence currents can be represented by vector rotating over rotor surface at $+6 \omega$ :

$$
F_{+6}\left(\theta_{r}, t\right)=\frac{3 n I_{+}}{\pi} \cos \left(6 \omega t+\phi_{+6}-\left(\theta_{r}-\delta\right)\right)
$$

and similarly for negative sequence currents we have MMF rotating at $-6 \omega$ :

$$
F_{-6}\left(\theta_{r}, t\right)=\frac{3 n I_{-}}{\pi} \cos \left(6 \omega t+\phi_{-6}+\left(\theta_{r}-\delta\right)\right)
$$

At $t=0$ the $F_{-6}$ has its maximum in $\theta_{r}=\delta-\varphi_{-6}$ and $F_{+6}$ at $\theta_{r}=\delta+\varphi_{+6}$ coinciding with disposition of, respectively, the $5^{\text {th }}$ and the $7^{\text {th }}$ stator related components (Fig. 1 ).

In case of equal amplitudes $I_{+}=I_{-}=I$ the resulted $6^{\text {th }}$ order MMF $F_{6 \_ \text {osc }}=F_{-6}+F_{+6}$ is an oscillating field distributed sinusoidally along air gap

$$
\begin{aligned}
& F_{-\cup+}(\theta) \\
& =\frac{6 n I}{\pi}\left[\cos \left(6 \omega t+\frac{\phi_{+6}+\phi_{-6}}{2}\right) \cdot \cos \left(\frac{\phi_{+6}-\phi_{-6}}{2}-\left(\theta_{r}-\delta\right)\right)\right](11) \\
& \text { with its amplitude fixed at angular ordinates } \\
& \theta_{r}=\left(\frac{\phi_{+6}-\phi_{-6}}{2}+\delta\right) \bmod \pi \text {. Here, any reference to harmonic }
\end{aligned}
$$


voltages on stator side disappears, as the relevant parameter of the MMF space distribution includes the difference $\left(\phi_{+6}-\phi_{-6}\right)$ rather than initial angles themselves. This phase difference will be noted $\phi_{6}$.

Theoretically, the phases $-\phi_{6}$ and $\phi_{+6}$ should both have the same values, encountering for the same harmonic impedances composed of PM's losses resistance and magnetization reactance, maybe also of leakage reactance. They cannot be different for equal MMFs rotating in opposite directions. Hence the value of $\phi_{6}$ is zero and the composed MMF on the Fig. 2 oscillates at leading angle $\delta$ in relation to rotor axe.

This angle is the key parameter of eddy current dissipation in rotor's magnets. Depending on its value, the losses superposition based on simulation of sliding fields may, or may not be pertinent.

As a matter of fact, losses generated by the oscillating field $F_{6 \_s s}$ depend on rotor's related position of its amplitude, more losses being dissipated when the corresponding vector oscillates in the middle of magnets' plate comparing to case when it oscillating between poles.

On the other hand, losses generated by any of the sliding fields $F_{-6}$ and $F_{+6}$ depend by no means on initial phase $\phi_{-6}$ and $\phi_{+6}$, and neither on angle $\delta$.

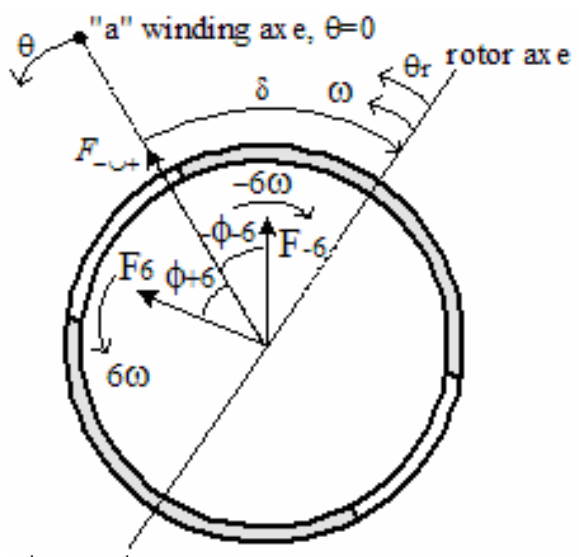

Fig. 2. The $-6^{\text {th }}$, the $+6^{\text {th }}$ MMF components and the resulting MMF in relation to rotor at $\mathrm{t}=0$.

With $5^{\text {th }}$ and $7^{\text {th }}$ current harmonics having different amplitudes, for example $\mathrm{I}_{-}>\mathrm{I}_{+}$the resulting MMF is composed of one oscillated and one sliding component

$$
\begin{aligned}
& F_{-\cup+}\left(\theta_{r}, t\right)= \\
& \frac{6 n I_{+}}{\pi}\left[\cos \left(6 \omega t+\frac{\phi_{+6}+\phi_{-6}}{2}\right) \cdot \cos \left(\frac{\phi_{+6}-\phi_{-6}}{2}-\left(\theta_{r}-\delta\right)\right)\right] \\
& +\frac{3 n\left(I_{-}-I_{+}\right)}{\pi} \cos \left(6 \omega t+\phi_{-6}+\left(\theta_{r}-\delta\right)\right)
\end{aligned}
$$

This case will be noted as mixed MMF. With the oscillating part growing, the dependence of losses on its angular position will grow. We will now establish the law concerning this dependence as well as conditions on PM loss superposition.

\section{PURELY OSCILLATING MMF}

\section{A. Surplus coefficient for losses and energies}

We assume eddy current dissipation as the only PM losses. Consequently, we take them as proportional to square of MMF, this proportionality being characterized by new loss coefficient $\mathrm{C}$ (13). For one sliding MMF it will be

$$
C=\frac{1}{T} \int_{0}^{T} \int_{\theta} F^{2} d \theta d t
$$

with $\theta$ - integration area of magnet's extent on rotor circumference.

In case where magnets cover two thirds of each pole we get for $F_{-6}$ and $F_{+6}$ the corresponding loss coefficients

$$
C_{-}=\frac{6 n^{2} I_{-}^{2}}{\pi} \text { and } C_{+}=\frac{6 n^{2} I_{+}^{2}}{\pi}
$$

Both are independent of the angle $\delta$. This will not be the case of the loss coefficient $C_{-\cup+}$ characterizing the oscillating MFF which is composed of $F_{-6}$ and $F_{+6}$. For equal amplitudes $I_{-}=I_{+}=I$ and $\phi_{6}=0$ we have

$$
C_{-\cup+}=\frac{12 n^{2} I^{2}}{\pi}+\frac{9 \sqrt{3} n^{2} I^{2}}{\pi^{2}} \cos (2 \delta)
$$

With these three coefficients we can define an analytic surplus parameter $\Delta_{a}$ which gives a measure of excess of losses calculated for oscillating MMF comparing to sum of losses of its two sliding components:

$$
\Delta_{a}=C_{-\cup+}-\left[C_{+}+C_{-}\right]=\frac{9 \sqrt{3} n^{2} I^{2}}{\pi^{2}} \cos (2 \delta)
$$

Except for two positions of the oscillating field, its losses don't equal the sum of losses and traditional superposition method will give erroneous results. In order to evaluate this error we introduce a relative surplus coefficient $\mathrm{SC}_{\mathrm{a}}$, with index a for "analytic":

$$
S C_{a}=\left[\frac{\left(\Delta_{a}\right)}{\left(C_{+}+C_{-}\right)}\right]_{I_{+}=I_{-}}=\frac{3 \sqrt{3}}{4 \pi} \cos (2 \delta)
$$

This analytic surplus coefficient reaches its maximum of $41.3 \%$ for $\delta=0^{\circ}$. Simulation of a FE machine model gives maximum of $36 \%$ occurring at $\delta=10^{\circ}$ (Fig. 3) 


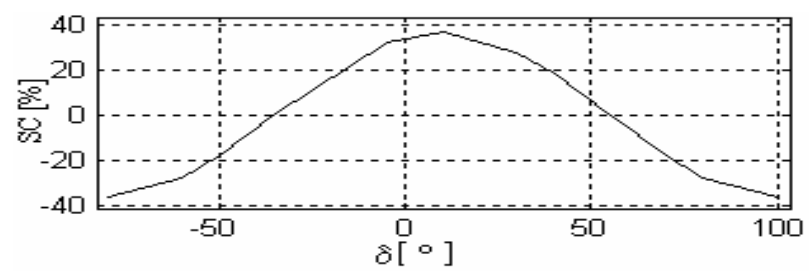

Fig. 3. FE simulated analytic loss surplus coefficient.

An analogous surplus coefficient can be defined for magnetic energies stocked in machine. Simulation confirms the efficiency rule (Fig. 4): with more losses we get less energy.

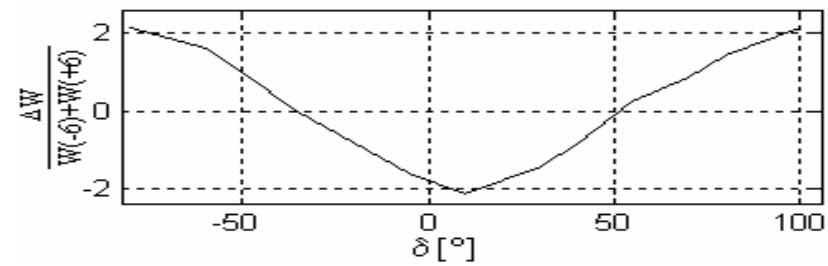

Fig. 4 FE simulated analytic surplus coefficient for magnetic energy

\section{MIXED MMF}

In case of different amplitudes of currents $I_{-}=I_{+}+\delta I$ the composite $\mathrm{MMF}$ is a sum of oscillating and sliding components. The corresponding loss coefficient can be represented as

$$
\begin{aligned}
C_{-\cup+}= & \frac{12 n^{2} I_{+}{ }^{2}}{\pi}+\frac{6 n^{2} \delta I^{2}}{\pi}+\frac{12 n^{2} I_{+} \delta I}{\pi}+ \\
& \frac{9 \sqrt{3} n^{2} I_{+}\left(I_{+}+\delta I\right)}{\pi^{2}} \cos (2 \delta)
\end{aligned}
$$

and the coefficients of individual losses are

$$
C_{+}+C_{-}=\frac{12 n^{2} I_{+}{ }^{2}}{\pi}+\frac{6 n^{2} \delta I^{2}}{\pi}+\frac{12 n^{2} I_{+} \delta I}{\pi}
$$

Then the analytical loss surplus becomes

$$
\Delta_{a}=C_{-\cup+}-\left[C_{+}+C_{-}\right]=\frac{9 \sqrt{3} n^{2}}{\pi^{2}}\left(I_{+}^{2}+I_{+} \delta I\right) \cos (2 \delta)
$$

As it was in the case of equal currents, $\Delta_{\mathrm{a}}$ is null for $\delta=$ $1+45^{\circ}$ and it is maximal for $\delta=0^{\circ}$. However, the ratio of the losses of the sum to the sum of losses $\mathrm{SC}_{\mathrm{a}}$ is lesser than for pure oscillating MMF:

$$
\begin{aligned}
S C_{a_{-} I_{+} \neq I} & =\left[\frac{\left(\Delta_{a}\right)}{\left(C_{+}+C_{-}\right)}\right]_{I_{+} \neq I_{-}}=\frac{3 \sqrt{3}}{4 \pi} \cos (2 \delta) \cdot k \\
& =C S_{a_{-} I_{+}=I_{-}} \cdot k
\end{aligned}
$$

with

$$
k=\frac{I_{+}^{2}+I_{+} \Delta I}{I_{+}^{2}+I_{+} \Delta I+0.5 \Delta I^{2}}=\frac{1}{1+0.5 \frac{\left(I_{-}-I_{+}\right)^{2}}{I_{+} I_{-}}}<1
$$

Its value less than 1 confirms theoretical consideration that the sliding component, present in the composite MFF, lowers the surplus coefficient. The equation (20) suggests also that any ratio $I / I_{+}$gives the same coefficient that its inverse (Fig. 6).

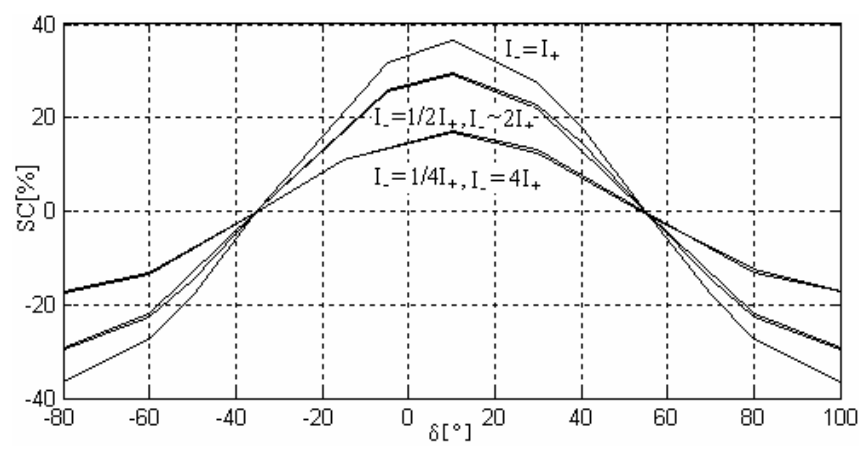

Fig. 5. Analytic loss surplus coefficient is the same for a given currents ratio and for its inverse. Simulation by FE.

Amplitude of the loss surplus SC varies between $12 \%$ and $25 \%$ for current ratio between 4 and 1 ; the error of superposition diminishes when the oscillating part of MMF becomes less. But obviously, when the part of sliding MMF is dominant, there's little to be superimposed.

\section{INFLUENCES OF LOAD}

Load depending currents moderate amplitudes of analytic surplus parameters $\Delta_{a}(16)$ and (19). Actually, currents rise with load, and so does the angle $\delta$. The most dissipating position of alternating flux corresponds also to low, no-load values of currents, whereas with $\delta$ near $90^{\circ}$, i.e. in low dissipating position, currents are high. Losses are obviously higher in low dissipation position.

This moderation doesn't change the relative surplus coefficients SC. Being here developed for the $5^{\text {th }}$ and the $7^{\text {th }}$ harmonics, it can be calculated for higher frequencies, like $11^{\text {th }}$ and $13^{\text {th }}, 19^{\text {th }}$ etc.

\section{CONCLUSION}

Losses superposition can deceive if adequate decomposition of field into sliding and oscillating fields is not operated. Error of losses estimation rises with square of turns' number in stator winding, and so it is more important for large machines.

The problem will disappear with future user-friendly FE packages permitting easy and efficient electro-dynamic modeling and simulation of rotor in movement and stator supplied with non sinusoidal voltage. This, however, doesn't seem to be near future. 


\section{REFERENCES}

[1] N Taghizadeh Irenji, S M Abu-Sharkh, M R Harris, "Effect of rotor sleeve conductivity on rotor eddy-current loss in high-speed PM machines", International Conference on Electrical Machines (ICEM2000), 2000, pp. 645-8.

[2] Naomitsu Usrasaki, Tomonobu Senjyu, Katsumi Uezato, "Relationship of parallel model and series model for permanent magnet synchronous moors taking iron loss into account", IEEE Trans. Energy Conversion, vol. 19 , no. 2, June 2004, pp. 265-270.

[3] James L. Kirtley Jr., "Loss models: Permanent Magnets", Massachusetts Institute of Technology - Department of Electrical Engineering and Computer Science, September 16, 1997

[4] James L. Kirtley Jr. ,"Eddy Currents, Surface Impedance and loss Mechanisms", Massachusetts Institute of Technology - Department of Electrical Engineering and Computer Science, February 11, 2004

[5] James L. Kirtley Jr. and Edward C. Lovelace, "Drag Loss in Retaining Rings of Permanent Magnet Motors", Electric Machines and Drives Conference, 2003(IEMDC'03), IEEE international, Vol 2, pp. 10681072, June, 2003

[6] H. Polinder and M. J. Hoeijmakers, "Eddy-current losses in the segmented surface-mounted magnets of a PM machine", IEE Pro.-Electr. Power Appl. Vol. 146, No. 3. May 1999

[7] J.-C.Vannier, R.Kaczmarek, Z.-Y.Wang, A.Randria, "Rotor Power Losses in a PM SM by Calculation, Simulation and Measurements, Mutual Verification of Methods", Proc. XLII Int. Symp. Electr. Mach. SME2006, pp. 35-38

\section{BIOGRAPHIES}

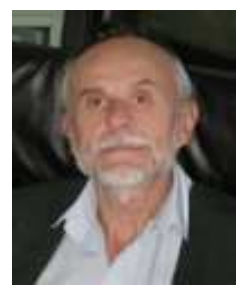

Robert Kaczmarek is professor in the Ecole Supérieure d'Electricité (Supelec) in France. His research interest is with power networks (fault detection, network protection, parameters identification), with magnetism (domain observation, loss prediction and measurements in soft and hard magnetic materials) and design of electric machines.

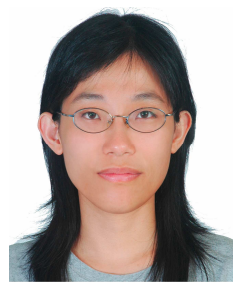

Wan-Ying HUANG has graduated from Tsing Hua University in Taiwan with Master Degree in 2002 and in 2006 has got her PHD in Ecole Supérieur d'Electricité SUPELEC and University Paris XI in France. She is now on a post doctoral research program in SUPELEC, working on protections and voltage stability in electrical systems as well as optimal design of electric machines.

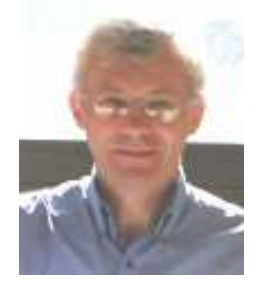

Jean-Claude Vannier is professor and Head of Department of Energy and Power Systems in the Ecole Supérieure d'Electricité (Supelec) in France. His research interest is with energy conversion systems (motors, actuators, generators) and concerns the modeling, the design and the optimization of these equipments for specific applications. 